\title{
Role of Mean Platelet Volume in the Prognosis of Locally Advanced Gastric Cancer: A Tertiary Cancer Center Experience
}

\author{
Manjunath $\mathrm{K} \mathrm{V}^{1}$, Pavan Jonnada ${ }^{1}$, Sai Kiran $\mathrm{N}^{2}$, Ali Anwar ${ }^{1}$ \\ 1. Surgical Oncology, Kidwai Memorial Institute of Oncology, Bangalore, IND 2. Surgical Oncology, Kidwai Memorial \\ Institute of Oncology, Bengaluru, IND
}

Corresponding author: Pavan Jonnada, pavan.j.1903@gmail.com

\section{Abstract \\ Introduction}

Mean platelet volume (MPV) is an inflammatory marker suggesting the activation of platelets. Many studies observed an association between MPV and cancer spread and metastasis. Hence, we have conducted a retrospective study to find the role of MPV as a prognostic marker in locally advanced gastric cancer.

\section{Materials and methods}

The present study included a retrospective review of 149 patients with gastric cancer who had neoadjuvant chemotherapy followed by surgery. MPV was obtained and then statistically analyzed to find an association between tumor $(\mathrm{T})$, node $(\mathrm{N})$, and overall stage as per the American Joint Committee on Cancer (AJCC) staging system, using Statistical Package for the Social Sciences (SPSS) software (IBM Corp., Armonk, NY).

\section{Results}

In our study, we observed that MPV values were significantly high in N+ disease (OR 3.794 (95\% CI 1.903 7.563); p-value 0.0001), higher T stage (OR for >T2 3.692 (95\% CI 1.876 - 7.266); p-value 0.0001), and advanced stage (OR 7.708 (95\% CI 3.258 - 18.237); p-value 0.0001) of gastric cancer.

\section{Conclusions}

MPV is an inflammatory marker that correlates with nodal disease and aids in the staging and prognostication of locally advanced gastric cancer. This inexpensive, convenient marker can aid in the risk stratification of locally advanced gastric cancer.

Received 06/30/2020 Review began 07/03/2020 Review ended 07/03/2020 Published 07/10/2020

(c) Copyright 2020 $\mathrm{K} V$ et al. This is an open access article distributed under the terms of the Creative Commons Attribution License CC-BY 4.0., which permits unrestricted use, distribution, and reproduction in any medium, provided the original author and source are credited.
Categories: Gastroenterology, General Surgery, Oncology

Keywords: mean platelet volume, gastric cancer, locally advanced, nodal disease, stage

\section{Introduction}

Gastric cancer is the fifth most common cancer and the third leading cause of cancer death [1]. The survival rates of Stages I, II, III, and IV were $80 \%-90 \%, 70 \%, 35 \%-54 \%$, and $16 \%$, respectively [2]. Despite improvements in screening and advancements in treatment, gastric cancer often presents at an advanced stage. The presentation of early gastric cancer at a stage at which it is resectable is observed in only $33 \%$ of cases [3]. Hence, it is crucial to diagnose the disease at an early stage, which can have a significant improvement in survival with timely treatment.

Several tumor markers have been used in the diagnosis, prognosis, and early detection of gastric cancer. The most frequent tumor markers used for the early detection of gastric cancer include carcinoembryonic antigen (CEA), the carbohydrate antigens (CAs) CA19-9, CA72-4, CA125, CA24-2, and CA50, and pepsinogen and $\alpha$-fetoprotein (AFP) [4]. Even so, the sensitivity of the markers used remains unsatisfactory and so far, none of them is unique for diagnostics or prognostic for gastric cancer [4-5]. Therefore, the identification of novel diagnostic and prognostic markers for gastric cancer is crucial.

Several factors, such as dietary, infectious, and genetic factors, are suspected to contribute to the development of gastric cancer [6]. Furthermore, in previous studies, it has been shown that gastric cancer represents an underlying inflammation-driven malignancy transformation [7].

Mean platelet volume (MPV) is a routinely determined parameter by complete blood count (CBC) analyzers [8]. It indicates the average size of platelets, the platelet production rate, and stimulation [9]. MPV has been revealed as a marker of inflammation in hepatocellular carcinoma and pancreatic adenocarcinoma [10]. 
The aim of this study is to examine whether MPV would be a useful inflammatory marker and assess whether MPV could predict nodal status and appropriate cut-off value. Besides, we investigated the relationship between MPV levels and cancer stage in gastric cancer patients.

\section{Materials And Methods}

We retrospectively investigated gastric cancer patients who underwent surgical resection at Kidwai Memorial Institute of Oncology between November 2017 and July 2019. Metastatic gastric cancer patients who underwent upfront surgery without neoadjuvant chemotherapy were excluded from the study. One-hundred forty-nine gastric cancer patients were included in the study. The staging of cancer was made according to tumor-node-metastases (TNM) classification and classified through the American Joint Committee on Cancer (AJCC) recommendations. The preoperative data were obtained from the recorded computerized database. The study was undertaken after institutional review board review clearance and with appropriate consent.

\section{Blood analysis}

About 5-7 ml, blood was collected from a peripheral vein and was collected into sterile ethylenediaminetetraacetic acid (EDTA) tubes. Blood samples were taken in the morning to minimize the impact of circulating hormones (circadian rhythm). Within 30 minutes after sample collection, an analysis of blood parameters was done by using a hematology analyzer. MPV was, therefore, obtained and used in the consequent analysis. The MPV chosen for the study is the MPV at the time of diagnosis.

The histopathological characteristics included the histological type and tumor grade. The laboratory variables were hemoglobin (g/fL) and hematocrit (\%),MPV (fL). Blood samples for laboratory analysis were been taken after eight to 12 hours of fasting.

\section{Statistical analysis}

Statistical analyses were performed with the Statistical Package for the Social Sciences (SPSS) software (IBM Corp., Armonk, NY). All parameters were expressed as means and standard deviation. The chi-square test was used to compare the categorical variables. The paired sample test was used to compare the preoperative and postoperative variables. A receiver-operating characteristic (ROC) curve analysis was performed to identify the optimal cut-off values of the MPV level. A p-value of less than 0.05 was considered statistically significant.

\section{Results}

We studied 149 patients of non-metastatic gastric cancer; there were 105 males and 44 females. The age of the patients was between 22 and 75 years and the mean age was 54.6. The baseline characteristics are presented in Table 1 . 


\section{Cureus}

Baseline characteristics of the study participants

Number of subjects

\section{Age range}

22 - 75 years

Mean age

54.6 years

Sex

Male

$106(28.9 \%)$

Female

$43(71.1 \%)$

Mean platelet volume - $\mathrm{fl}$ (mean)

$9.87 \mathrm{fL}$

TNM staging

Stage 1

Stage 2

56

Stage 3

Histology

Intestinal

Diffuse

31

\section{TABLE 1: Baseline demographic characteristics}

TNM: tumor-node-metastases

Laboratory tests showed that gastric cancer patients enrolled in this study had MPV values ranging between 7.8 and $11.5 \mathrm{fl}$; the mean MPV value was $9.87 \mathrm{fl}$.

ROC curves were constructed to allot optimal cut-off values of MPV associated with lymph node positivity in gastric cancer patients. Results were significant ( $p$-value $=0.0001$ ). The area under the curve was 0.720 for MPV (Figure 1). The analysis showed that MPV higher than 10.25 indicates the positivity of lymph nodes with $62 \%$ sensitivity and $80 \%$ specificity. 


\section{Cureus}

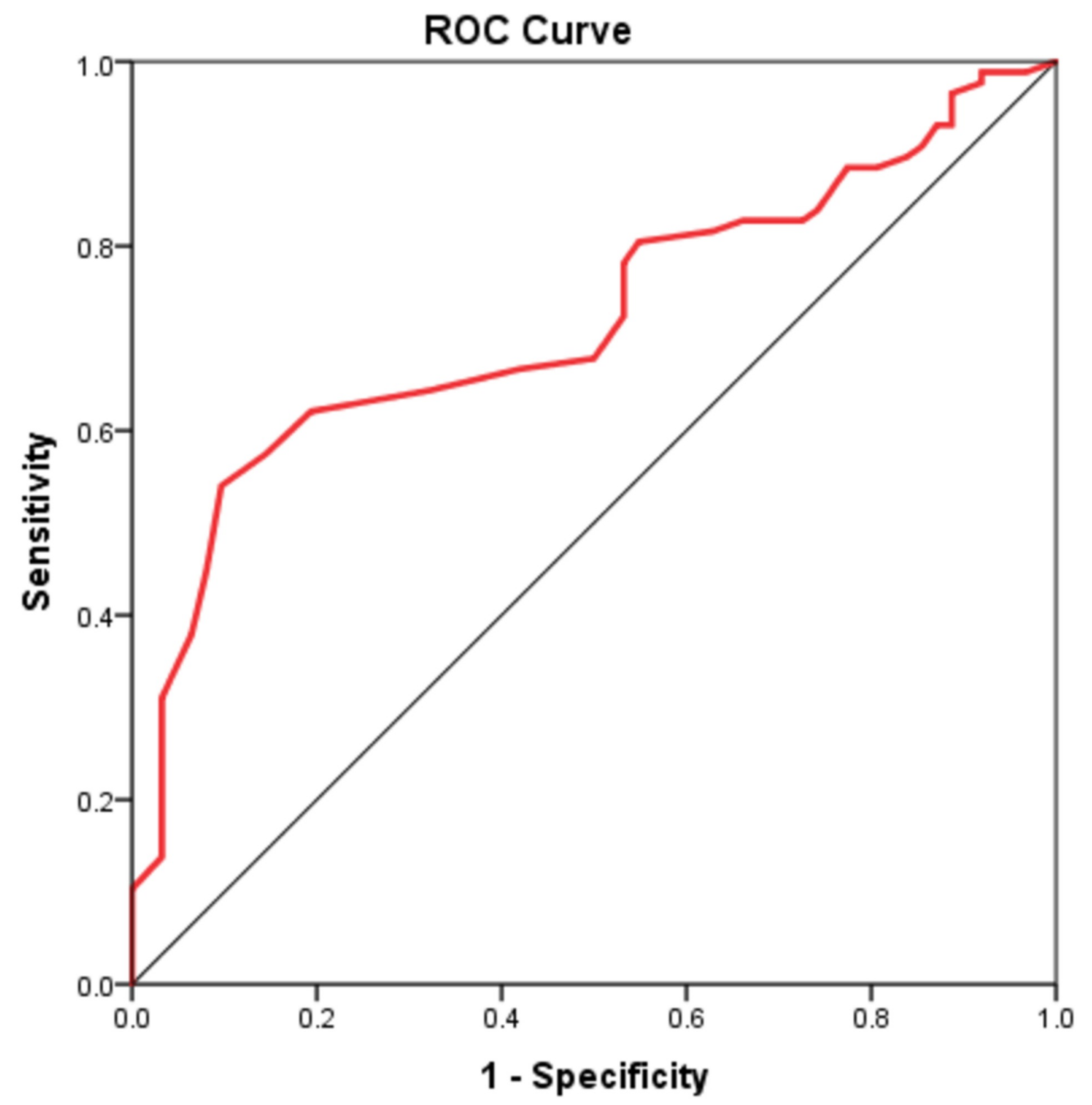

Area Under Curve $=0.720$

FIGURE 1: ROC curve analysis showing MPV cut-off

ROC: receiver operating characteristic; MPV: mean platelet volume

The patients were separated into two groups according to median preoperative MPV: low $(<10.2)$ MPV and high ( $\geqslant 10.2)$ MPV. There was no significant association was found between high MPV values and sex (OR 0.8 (95\% CI 0.428 - 1.769) p-value - .700). Advanced age was not found to be associated with increasing MPV (OR 1.006 (95\% CI 0.517-1.958) p-value .98). We observed that greater MPV values were associated with a higher T stage (OR 3.692 (95\% CI 1.876- 7.266) p-value .0001) as shown in Figure 2, with a higher N stage (OR 3.794 (95\% CI 1.903 - 7.563) p-value .0001) as shown in Figure 3 and with advanced stages (OR 7.708 (95\% CI 3.258- 18.237) p-value .0001), as shown in Figure 4. There was a significant negative association noted between elevated MPV and the diffuse type of histology of gastric cancer (OR 0.345 (95\% CI 0.147 0.812) p-value .012), as shown in Table 2. 


\section{Cureus}

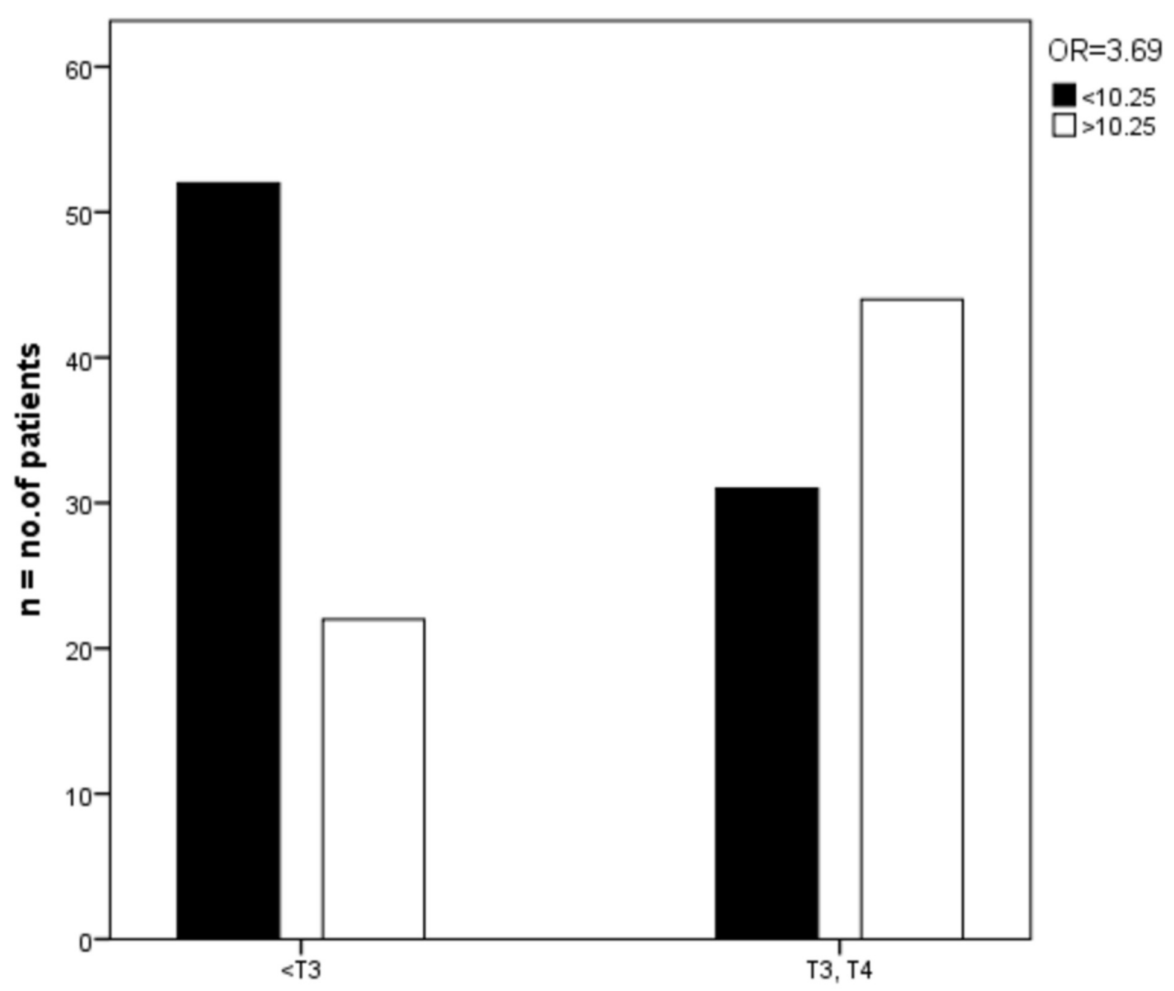

Figure 3: Relation between T stage and MPV

FIGURE 2: Relation between T stage and MPV

T: tumor; MPV: mean platelet volume

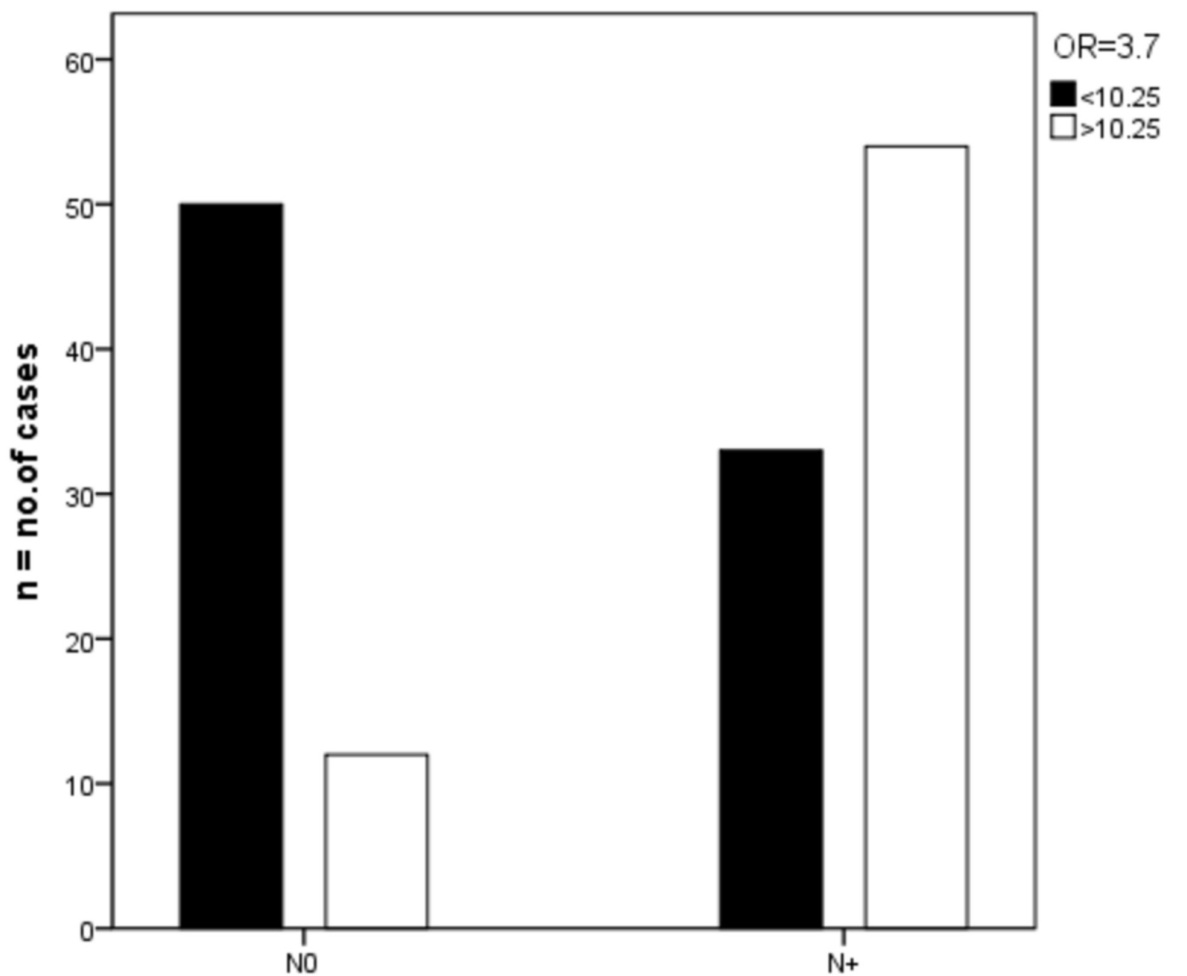

Figure 3: Relation between $\mathrm{N}$ stage and MPV

FIGURE 3: Relation between N stage and MPV 


\section{Cureus}

$\mathrm{N}$ : node; MPV: mean platelet volume

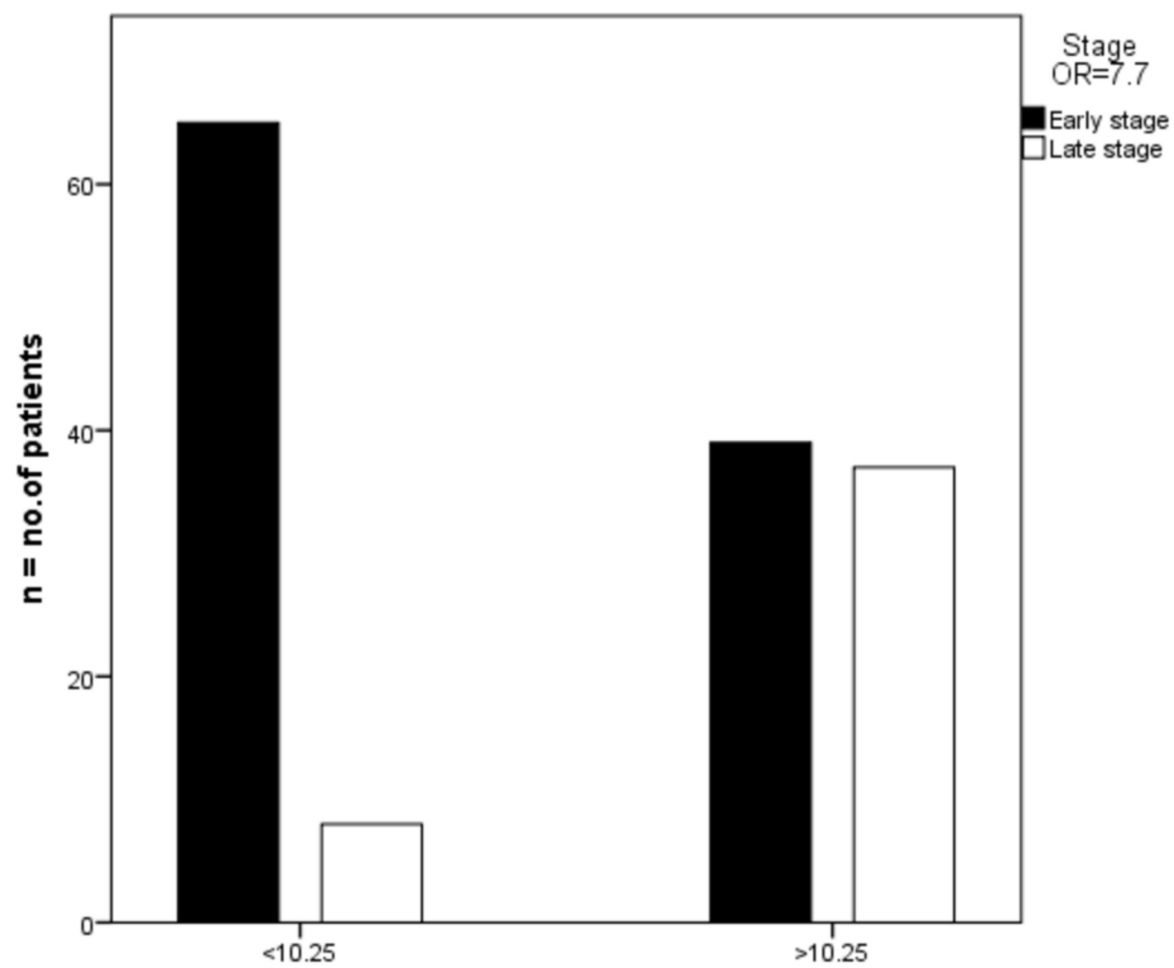

Figure 4: Relation of MPV and Stage

FIGURE 4: Relation between stage and MPV

MPV: mean platelet volume 


\section{Cureus}

\begin{tabular}{|c|c|c|c|c|c|c|}
\hline & No.of patients & Low MPV (<10.2)(n) & High MPV (>10.2)(n) & Chi square & p-value & Odds Ratio (95\% Cl) \\
\hline Gender & & & & 0.149 & .700 & $0.8(0.42-1.76)$ \\
\hline Male & 106 & 53 & 53 & & & \\
\hline Female & 43 & 20 & 23 & & & \\
\hline Age & & & & .0001 & 0.985 & $1.006(0.51-1.95)$ \\
\hline$<50$ years & 55 & 27 & 28 & & & \\
\hline$>50$ years & 94 & 46 & 48 & & & \\
\hline T stage & & & & 14.819 & .0001 & $3.962(1.87-7.26)$ \\
\hline T1, T2 & 74 & 48 & 26 & & & \\
\hline 13,14 & 75 & 25 & 50 & & & \\
\hline N stage & & & & 14.936 & .0001 & $3.794(1.90-7.56)$ \\
\hline No & 62 & 42 & 20 & & & \\
\hline$N+$ & 87 & 31 & 56 & & & \\
\hline Histology & & & & 6.241 & .012 & $0.345(0.14-0.81)$ \\
\hline Intestinal & 118 & 64 & 54 & & & \\
\hline Diffuse & 31 & 9 & 22 & & & \\
\hline Stage & & & & 25.139 & .0001 & $7.708(3.25-18.23)$ \\
\hline Stage I, II & 104 & 65 & 39 & & & \\
\hline Stage III & 45 & 8 & 37 & & & \\
\hline
\end{tabular}

\section{TABLE 2: Relationship between MPV and demographic and clinical parameters}

T: tumor; N: node; MPV: mean platelet volume

\section{Discussion}

The present study established the role of MPV as an indicator of the status of nodal disease and, thereby, the prognosis of locally advanced resectable gastric cancer. Our results show that MPV can be a future prognostic marker that can aid in detecting the presence of nodal disease and correlate with the T-N staging of the AJCC staging system and, hence, over all the stages of the disease.

MPV increases with the activation or hyper-aggregation of platelets, reflecting the functional state and volume of platelets in the blood circulation. MPV serves as a negative or positive acute-phase reactant and is a marker of inflammation [11]. Cancer-associated inflammation is involved in the development and dissemination of multiple types of tumors [12]. The cascade responsible for the occurrence of cancer involves mainly two steps. First, tumorigenesis is mainly due to deoxyribonucleic acid (DNA) damage and defects in the repair mechanism by the production of reactive oxygen species (ROS) by inflammatory cells. Second, inflammatory cells produce cytokines, chemokines, and cell adhesion molecules, which are responsible for metastasis [13]. Several studies have confirmed the role of MPV in the inflammatory cascade, and the study by Khode et al. showed that high MPV levels are associated with a high risk of acute myocardial infarction when compared with healthy controls [14].

The elevation of MPV gained significance in malignancies as reported in several studies. Li et al. suggested that higher MPV levels are significantly observed in colon cancer patients when compared to healthy controls [15]. Kilincalp et al. suggested that MPV could be used as an early marker of the diagnosis of gastric cancer and can be used to monitor healthy subjects. Their study also showed that MPV levels were markedly raised in gastric cancer patients [16]. However, the study by Zhi-Yuan Yun et al. contradicted these findings, raising doubts regarding the role of MPV as an early diagnostic marker in gastric cancer patients [17].

Sun SY et al. suggested that low MPV was significantly associated with locally advanced esophageal carcinoma and may serve in the screening and risk stratification of locally advanced esophageal carcinomas [18]. Our study showed that increased MPV is significantly associated with a higher T and N stage and, 
thereby, might be helpful in risk stratification of the disease. Shen et al. showed that higher preoperative MPV values were significantly associated with lower survival rates for patients of resectable gastric cancer patients [19]. We investigated whether higher MPV could assess the nodal disease and, to our knowledge, this is the first study demonstrating the role of MPV as a maker of locally advanced disease.

Various studies have used different cut-offs for MPV in various cancers. In our study, an MPV cut-off of 10.25 indicated the positivity of lymph nodes with $62 \%$ sensitivity and $80 \%$ specificity based on the ROC analysis. A similar level of cut-off was used in other contemporary studies on gastric cancer [19].

Our study has its limitations, as it is a retrospective series based on case records. A major drawback of this investigation is the limited detail available for each case. Furthermore, all patients underwent neoadjuvant chemotherapy and those with a favorable response underwent surgery. This may indicate the favorable biology of the tumors and may not completely reflect the role of MPV.

However, the present study indicates an association of the MPV level and with the local invasion and prognosis of gastric cancer. Even though MPV is also a non-specific marker, this non-invasive, wellsituated, and inexpensive biomarker may be an addition to the present biomarkers and a benefit to the prognosis and risk stratification of locally advanced gastric cancer.

\section{Conclusions}

Mean platelet volume is an inexpensive, convenient marker that correlates with nodal disease and aids in staging, prognostication, and risk stratification of locally advanced gastric cancer. The MPV cut-off value of 10.25 predicts nodal metastases and a higher $\mathrm{T}$ stage and thus provides the stage of the disease. The incorporation of MPV into current prognostic factors may provide additional information to stratify the disease and may provide an opportunity for further investigation into the natural history and prognosis of the disease.

\section{Additional Information \\ Disclosures}

Human subjects: Consent was obtained by all participants in this study. Animal subjects: All authors have confirmed that this study did not involve animal subjects or tissue. Conflicts of interest: In compliance with the ICMJE uniform disclosure form, all authors declare the following: Payment/services info: All authors have declared that no financial support was received from any organization for the submitted work. Financial relationships: All authors have declared that they have no financial relationships at present or within the previous three years with any organizations that might have an interest in the submitted work. Other relationships: All authors have declared that there are no other relationships or activities that could appear to have influenced the submitted work.

\section{References}

1. Bray F, Ferlay J, Soerjomataram I, Siegel RL, Torre LA, Jemal A: Global cancer statistics 2018: GLOBOCAN estimates of incidence and mortality worldwide for 36 cancers in 185 countries. CA Cancer J Clin. 2018, 68:394-424. 10.3322/caac.21492

2. Katai H, Ishikawa T, Akazawa K, et al.: Five-year survival analysis of surgically resected gastric cancer cases in Japan: a retrospective analysis of more than 100,000 patients from the nationwide registry of the Japanese Gastric Cancer Association (2001-2007). Gastric Cancer. 2018, 21:144-154. 10.1007/s10120-0170716-7

3. Afridi SP, Bano F, Shafiq-ur-Rahman: Pattern and presentation of carcinoma stomach. J Coll Physicians Surg Pak. 2011, 21:161-163.

4. Tsai MM, Wang CS, Tsai CY, et al.: Potential diagnostic, prognostic and therapeutic targets of MicroRNAs in human gastric cancer. Int J Mol Sci. 2016, 17:945. 10.3390/ijms17060945

5. Tong W, Ye F, He L, et al.: Serum biomarker panels for diagnosis of gastric cancer . Onco Targets Ther. 2016, 2016:2455-2463. 10.2147/OTT.S86139

6. Hundahl SA, Phillips JL, Menck HR: The National Cancer Data Base Report on poor survival of U.S. gastric carcinoma patients treated with gastrectomy: Fifth Edition American Joint Committee on Cancer Staging, proximal disease, and the "different disease" hypothesis. Cancer. 2000, 88:921-932.

7. Kim DK, Oh SY, Kwon HC, et al.: Clinical significances of preoperative serum interleukin- 6 and C-reactive protein level in operable gastric cancer. BMC Cancer. 2009, 9:155. 10.1186/1471-2407-9-155

8. Bath PM, Butterworth RJ: Platelet size: measurement, physiology and vascular disease . Blood Coagul Fibrinolysis. 1996, 7:157-161.

9. Kai H, Kitadai Y, Kodama M, et al.: Involvement of proinflammatory cytokines IL-1beta and IL-6 in progression of human gastric carcinoma. Anticancer Res. 2005, 25:709-713.

10. Kurt M, Onal IK, Sayilir AY, et al.: The role of mean platelet volume in the diagnosis of hepatocellular carcinoma in patients with chronic liver disease. Hepatogastroenterology. 2012, 59:1580-1582.

11. Biricik S, Narcı H, Dündar GA, Ayrık C, Türkmenoğlu MÖ: Mean platelet volume and the ratio of mean platelet volume to platelet count in the diagnosis of acute appendicitis. Am J Emerg Med. 2019, 37:411-414. 10.1016/j.ajem.2018.05.075

12. Şahin F, Aslan AF: Relationship between inflammatory and biological markers and lung cancer. J Clin Med. 


\section{Cureus}

2018, $7: 160.10 .3390 / j \mathrm{~cm} 7070160$

13. Maletzki C, Emmrich J: Inflammation and immunity in the tumor environment. Dig Dis. 2010, 28:574-578. $10.1159 / 000321062$

14. Khode V, Sindhur J, Kanbur D, Ruikar K, Nallulwar S: Mean platelet volume and other platelet volume indices in patients with stable coronary artery disease and acute myocardial infarction: a case control study. J Cardiovasc Dis Res. 2012, 3:272-275. 10.4103/0975-3583.102694

15. Li JY, Li Y, Jiang Z, Wang RT, Wang XS: Elevated mean platelet volume is associated with presence of colon cancer. Asian Pac J Cancer Prev. 2014, 15:10501-10504. 10.7314/apjcp.2014.15.23.10501

16. Kılınçalp S, Ekiz F, Başar O, et al.: Mean platelet volume could be possible biomarker in early diagnosis and monitoring of gastric cancer. Platelets. 2014, 25:592-594. 10.3109/09537104.2013.783689

17. Yun ZY, Li N, Zhang X, et al.: Mean platelet volume, platelet distribution width and carcinoembryonic antigen to discriminate gastric cancer from gastric ulcer. Oncotarget. 2017, 8:62600-62605. 10.18632/oncotarget.15898

18. Sun SY, Zhao BQ, Wang J, Mo Z-X, Zhao Y-N, Wang Y, He J: The clinical implications of mean platelet volume and mean platelet volume/platelet count ratio in locally advanced esophageal squamous cell carcinoma. Dis Esophagus. 2018, 31:dox125. 10.1093/dote/dox125

19. Shen XM, Xia YY, Lian L, et al.: Mean platelet volume provides beneficial diagnostic and prognostic information for patients with resectable gastric cancer. Oncol Lett. 2016, 12:2501-2506. 10.3892/ol.2016.4913 